\title{
Estimación de Parámetros de Estabilidad para Determinar la Respuesta de Híbridos de Maíz (Zea mays L.) a Ambientes Contrastantes de Centro América, Panamá y Mexico1
}

\author{
Hugo S. Córdova ${ }^{2}$
}

\section{COMPENDIO}

La interacción genotipo-ambiente merece gran importancia en la evaluación de cultivares desarrollados para diferentes ambientes de producción, las diferencias entre ambientes y años pueden cambiar la magnitud de la respuesta relativa de los cultivares a ambientes contrastantes. Por esta razón, es necesario la integración de los conceptos de estabilidad para definir la adaptación de cultivares. El objetivo del presente trabajo es determinar la adaptación de los híbridos de maíz desarrollados por los programas nacionales y compañías privadas a las diferentes regiones maiceras de Centro América y El Caribe. Treinta y seis híbridos fueron evaluados a través de 15 ambientes de Norte, Centro América y Panamá. Los modelos AMMI y Eberhart y Russell se utilizaron para estimar la estabilidad de los cult $\sim$ vares.

El nuevo híbrido HB-85 de ICTA, Guatemala rindió $5.700 \mathrm{~kg} / \mathrm{ha}$ de grano superando al testigo $\mathrm{H}-5$ con un $34 \%$ y con excelente calidad de mazorca (cobertura y pudrición)su adaptación fue sobresaliente a la mayoría de ambientes favorables y desfavorables, en forma similar se comporto el híbrido P-8802 de Panamá. El modelo AMMI identificó a los híbridos MAX-309, P-8812, P-8822, HB-85 y HB-87 como los más estables. Los híbridos HB-85, HB-87 y HB-83 M mostraron estabilidad del rendimiento $\left(\beta_{1}=1, S_{\mathrm{di}}=0\right)$ a través de 34 ambientes y 2 años. Se ha realizado notable progreso en la formación de híbridos en la región de Centro América y El Caribe, seleccionando en contra de factores adversos bióticos y abióticos. Se sugiere la promoción de los nuevos cultivares para incrementar la productividad del cultivo del maíz en la región.

Palabras claves adicionales: genotipo-ambiente, Genotipos, interacción.

\section{INTRODUCCIÓN}

La interacción genotipo-ambiente merece gran importancia en la evaluación de cuItivares desarrollados para diferentes circunstancias de producción, es necesario la integración de los conceptos de estabilidad para definir el comportamiento de cultivares evaluados a través de ambientes constrastantes. Los métodos de evaluación de cultivares, en los cuales se realiza una descomposición de la interacción genotipo - ambiente, es muy útil para identificar genotipos estables (Tai, 1971) citado por Shukla (1972). La interacción genotipo - ambiente encontrada en estudios de evaluación de cultivares en varios ambientes justifica la integración del rendimiento y estabilidad del comportamiento (Kang et al. 1990), Kang (1988 B) desarrolló un método que

\begin{abstract}
3
Genotype by environment interactions are extremely important when evaluating cultivars developed for diverse environmental conditions. Differences in environments and years can change the relative ranking of genotypes across environments. Thus, it is necessary to incorporatestability parameters to properly define the adaptation ofcultivars to given environments. The objective of this study was to determine the adaptation of maize hybrids developed by National Programs and private seed industries in Central Aroerica and the Caribbean. Thirty six (36) hybrids were evaluated across 151ocations during 1989 throughout North and Central America. Cultivar, performance and yield stability was analyzed using AMMI and Eberhart and Russell models.

Hybrid HB-85 from ICTA yielded $5.7 \mathrm{t} / \mathrm{ha}$, outyielding the local check $\mathrm{H}-5$ by $34 \%$. In addition, this hybrid had excellent husk cover and minimal ear rot, and proved adapted to both favorable and unfavorable environments. Hybrid P-8802 from Panama also showed similar performance. The AMMI model identified the hybrids MAX-309, P-8812, P-88222, HB-85 and HB-87 as the most stable. Hybrids HB-85, HB-87, and HB-83M showed stability of grain yield $\left(\beta_{1}=1, S_{\mathrm{di}}=0\right)$ across 34 sites and 2 years. Good progress has been achieved in the production of hybrids adapted to adverse abiotic and biotic stresses for the Central American and Caribbean region. It is sllggested that the use ofthese new hybrids be encouraged to increase the productivity of maize in the region.
\end{abstract}

integra la media de rendimiento y un parámetro de estabilidad genética $\left(\sigma_{\mathrm{i}}^{2}\right)$ de Shukla (1972).

Una evaluación realista del comportamiento de cultivares adaptados a ambientes pobres y ricos, debe involucrar localidades cuya magnitud de la incidencia de factores adversos bióticos y abióticos contribuya a reducir la producción. La aplicación de modelos donde se estiman parámetros de estabilidad que identifican el comportamiento de los cultivares a través de diversos ambientes, contribuyen a la selección apropiada de los genotipos (Córdova 1989). El mejoramiento para adaptación amplia puede proveer la identificación de genotipos cuyo comportamiento sea superior en ambientes pobres

\footnotetext{
1 Trabajo presentado en la XXXVI Reunión Anual del PCCMCA celebrada en San Salvador, El Salvador del 26 al 30 de marzo de 1990.

2 Representante Regional del Programa de Maíz de CIMMYT para Centro América y El Caribe.

${ }^{3}$ El abstract es traducción del compendio.

Publicado en Agronomía Mesoamericana. Vol. 2 (1991).
} 
y ricos, estos ambientes pueden estar caracterizados por condiciones de estrés, un cultivar estable bajoestas circunstancias poseed una baja, interacción genotipo por ambiente, un coeficiente de regresion cercano $1.0 \mathrm{y}$ un alto rendimiento. La importancia que tienen los factores responsables de la estabilidad del rendimiento a través de ambientes contrastantes, y la identificación de esos factores específicos es muy relevante para entender la naturaleza de la estabilidad del rendimiento y puede abrir un camino para el desarrollo de criterios de selección adicionales al rendimiento (Blum 1988).

El principal objetivo en el mejoramiento del maíz es la estabilidad del rendimiento o la respuesta consistente a condiciones óptimas y subóptimas. Recientemente los científicos preocupados por la producción de alimentos en el mundo, hacen esfuerzos por obtener progresos en rendimiento que sean sostenidos y duraderos. Este nuevo concepto solo se puede lograr a través del desarrollo de germoplasma, cuya respuesta sea consistente a través de ambientes marginales y bajo presión de factores limitantes Bióticos y Abióticos que respondan positivamente a ambientes favorables. Cuando una serie de genotipos se evaluan a través de años y localidades, el ambiente consiste en numerosos factores físicos, químicos y biológicos actuando independiente e interactuando entre ellos.

Yates y Cochran, Citados por Blum (1988); Finlay y Wilkinson (1963); Eberhart y Russell (1966) utilizaron modelos de regresión e índices ambientales y establecieron parámetros de estabilidad para determinar el comportamiento de cultivares a través de una serie de ambientes contrastantes. Genotipos con una media de rendimiento alta, $\beta_{1}=1, \mathrm{~S}_{\mathrm{di}}$ $=0$ son definidos como estables en la concepción del último de los autores. Durante los últimos 10 afios estos modelos han sido utilizados ampliamente en Centro América y El Caribe (Córdova, 1988).

El modelo AMMI (Efectos principales aditivos e interacción multiplicativas), es el modelo a escoger cuando existen datos de experimentos que muestran significancia en los efectos principales y sus interacciones, AMMI integra algunos modelos estadísticos comúnmente aplicados a series de ensayos de rendimiento, esta integración incluye el análisis de varianza (ANV A) cuyo modelo es aditivo, el análisis de componentes principales (PCA) el cual es multiplicativo y el modelo de regresión lineal de Pinlay-Wilkinson (Zobel y Gauch, 1989).

Este trabajo tiene los siguiente objetivos:

a. Determinar la adaptación de los híbridos de Maíz desarrollados por los Programas Nacionales y Compañías Privadas a las diferentes regiones maiceras de Centro América y El Caribe.

b. Estimar los parámetros de estabilidad que permiten describir los genotipos de acuerdo a su respuesta a través de ambientes contrastantes.

c. Realizar un análisis combinado de años y localidades a través de un modelo comprensible que permita inferencias acertadas.

d. Establecer un mecanismo oficial en el cual los programas nacionales puedan basar sus decisiones en cuanto a la selección del germoplasma adecuado a las circunstancias de cada país, de tal manera que la información de varias localidades y años analizada en forma combinada genere recomendaciones más confiables.

\section{REVISIÓN DE LITERATURA}

Las pruebas de comportamiento de variedades cuando se realizan convencionalmente ofrecen información sobre la interacción genotipo - ambiente, pero no dan una idea de la estabilidad de las variedades evaluadas (Córdova et al. 1978). De allí que el análisis de estabilidad, es un buen instrumento en la identificación de germoplasma de gran potencial para los programas de mejoramiento. En base a la interpretación de los parámetros de estabilidad, Carballo y Márquez(1970) clasifican a una variedad estable cuando $\beta_{1}=$ $1, \mathrm{~S}_{\mathrm{di}}=0$, además por tener una alta media de rendimiento en relación con el resto de variedades.

Sprague y Jenkins (1943) y Allard yBradshaw (1967), citados por CÓrdova (1977), coinciden en que la mayor diversidad genética (cruzas simples en maíz por ejemplo) dota a las poblaciones de mayor estabilidad, que las hace idóneas para utilizarse también en ambientes desfavorables.

Allard y Bradshaw (1967), describen dos formas a través de las cuales una variedad puede exhibir estabilidad: 1. amortiguamiento poblacional; la variedad puede estar constituída de varios genotipos cada uno adaptado a un rango de ambientes un tanto diferentes y 2 . amortiguamiento individual; los individuos mismos pueden tener también amortiguamiento de manera que cada miembro de la población esté bien adaptado a un amplio rango de condiciones ambientales. De esta forma, las poblaciones homogéneas: homocigóticas o heterocigóticas (líneas puras y cruzas simples, respectivamente) dependerán obviamente del amortiguamiento individual para tener una población estable, mientras tanto el amortiguamiento individual como el poblacional podrán estar presentes en poblaciones heterogéneas. El amortiguamiento poblacional, se refiere a aquel que se encuentra por arriba de los constituyentes de la población, por lo que resulta de las interacciones entre los diferentes genotipos que coexisten en ella. Citan como ejemplo la revisión hecha por Simonds (1964), quien encontró que poblaciones mezcladas son casi siempre más estables en rendimiento que sus componentes individuales, y el trabajo de Jones (1958), que compara cruzas simples y dobles, encontran- 
do que los coeficientes de variación fueron menores en las cruzas dobles $(12.31 \%)$ que para cruzas simples $(21.41 \%)$.

El progreso en rendimiento y adaptabilidad se podría lograr fácilmente si se identifican genotipos estables en generaciones tempranas (Bonny y Kerlova, 1985).

Eberhart y Russell (1966) citados por Córdova (1978) postulan que aunque la estabilidad de una cruza doble proviene de la mezcla de genotipos, también parece que está bajo control genético o sea que ciertos genotipos pueden mostrar mayor estabilidad que otros, de manera que pueden obtenerse cruzas simples, genéticamente estables de mayor rendimiento que las cruzas dobles. En su investigación encontraron cruzas simples tan estables como cualquier cruza doble, sugiriendo que las cruzas simples difieren en su habilidad de respuestas a condiciones ambientales más favorables; la suma de cuadrados de desviaciones de regresión parece ser el parámetro más importante y que es probable que estén involucrados en esa estabilidad todos los tipos de acción genética.

Carballo y Márquez (1970), en su trabajo sobre estimación de parámetros de estabilidad en variedades de maíz, hacen notar que el grupo de variedades de alto rendimiento, los coeficientes $\beta_{\mathrm{i}}$ no difieren mucho de 1 o son inferiores a este valor. Mencionan que la tendencia general fue la asociación de altos rendimientos con altos valores de $\beta_{\mathrm{i}}$ y la asociación negativa de rendimiento y de $\beta_{\mathrm{i}}$ con $\mathrm{S}^{2} \mathrm{~d}_{\mathrm{i}}$.

Gardner y Mareck (1977), en su trabajo de evaluación de cuatro poblaciones formadas a través de selección masal, su variedad progenitora Hays y Golden y un híbrido testigo sembrados en catorce localidades, calcularon parámetros de estabilidad para cada entrada, utilizando la regresión para rendimiento con un índice ambiental, las poblaciones seleccionadas mostraron una respuesta mayor $\left(b_{i}=1.01\right.$ a 1.30$)$. El híbrido testigo $\left(b_{i}=0.74\right)$ en todas las localidades obtuvo rendimientos bajos, los rendimientos de la población seleccionada no fueron diferentes a los de la población original, concluyeron que la selección ha sido eficiente para aquellos alelos que permiten a las poblaciones mejorar o responder a las prácticas modernas de cultivo. La respuesta estimada a la selección variará dependiendo del nivel de rendimiento de la localidad en prueba. Esto explica el porqué en evaluaciones de la respuesta de la selección en masa en años secos (1974, 1975 y 1976) encontraron un decremento en rendimientos relativos de poblaciones mejoradas, en comparación con las poblaciones originales. Miezan et al. (1977), mencionan una expansión de la fórmula de coeficiente de regresión sugerida por Finlay y Wilkison, (1963) como parámetro de estabilidad, demostrando que el parámetro puede ser significativamente alterado por genotipos extremos, ejemplo: aquellos con una varianza pequeña o muy grande. Al parecer, no todos los genotipos deberán involucrarse en la estimación de índices ambientales.

Dos métodos han sido sugeridos: (1) Utilizar un juego de genotipos de igual varianza (dentro de las magnitudes in- termedias) para estimar los índices ambientales; (2) Usar la media ajustada de los genotipos en cada ambiente para definir el ambiente. Ellos utilizaron los datos de rendimiento de maíz en Kansas como ejemplo, confirmando los efectos de los genotipos externos. Sin embargo, no se obtuvieron cambios significativos en el coeficiente (sw) de regresión cuando se usaron diferentes combinaciones de genotipos para estimar los índices ambientales. Encontraron dificultad en la interpretación del coeficiente de regresión cuando la varianza entre genotipos no es cero.

Rows y Andrews (1974), citados por Córdova' 0978) estudiaron la estabilidad de seis poblaciones de maíz representativas de cuatro grados de heterocigocidad: líneas endogámicas $(90 \%) \mathrm{F}_{3}$ y $\mathrm{RC}_{2}(25 \%), \mathrm{F}_{2}$ y $\mathrm{RC}_{1}(50 \%)$ y $\mathrm{F}_{1}$ (100\%). Para el carácter rendimiento, tomando ala componente de varianza entre ambientes como criterio (F 2) encontraron asociado un mayor grado de heterocigocidad con tamaños mayores de 2, o sea con una menor estabilidad. En relación a la diversidad genética, los autores encontraron "Sorpresivo" que las poblaciones $\mathrm{F}_{2}, \mathrm{~F}_{3}$ y $\mathrm{RC}_{2}$ (heterogéneas) no fueron más estables que la del grupo de líneas (homogéneas) añadiendo que las $F_{1}$ deberían haber sido mas estables que las líneas. Con respecto a la componente VE no hubo una asociación clara con el nivel de heterocigocidad, presentándose descendentemente su tamaño como sigue: Líneas $\mathrm{F}_{1}, \mathrm{~F}_{3}, \mathrm{RC}_{2}$ y $\mathrm{F}_{2}$, o sea que las líneas y las $\mathrm{F}_{1}$ interaccionaron mas con los ambientes. En el análisis de regresión de cada grupo sobre los ambientes, de acuerdo al método de Finlay y Wilkison (1963), los mayores cuadrados medios para las desviaciones de regresión correspondieron también a las líneas ya lasF $\mathrm{F}_{1}$, mientras que los coeficientes de regresión aumentaron con mayor grado de heterocigocidad.

Salguero et al. (1977) evaluaron diez variedades e híbridos de maíz en once ambientes en Sur-Oriente de Guatemala, encontrando variedades estables $\left(\beta_{\mathrm{i}}=1\right)\left(\mathrm{S}_{\mathrm{di}}{ }^{2}=0\right)$, los cuales tuvieron altos rendimientos aún bajo condiciones de humedad limitada.

Dávila et al. (1978) estimaron los parámetros de estabilidad utilizando el modelo de Eberhart y Russell (1966), para identificar germoplasma criollo utilizable en el Programa de Mejoramiento del Altiplano, alto y medio. Los autores concluyen que dentro del germoplasma criollo existen variedades con alto potencial de rendimiento y estabilidad mostrada a través de nueve localidades del altiplano medio de Guatemala. A la vez encontraron que altos rendimientos están positivamente correlacionados a coeficientes de regresión y desviaciones de regresión $(r=0.99$ y 0.66 , respectivamente).

De paz et al. (1977-1978), encontraron una fuerte interacción entre variedad por ambiente al evaluar variedades mejoradas y criollas en el altiplano de Guatemala. Por lo que recomiendan que la estabilidad se puede mejorar evaluando las familias (que constituyen las poblaciones, que darán origen a. 
Córdova et al. (1978, 1988), utilizando el modelo de Eberbart y Russell para determinar el comportamiento de cultivares a través de varios afios y localidades, encontró respuestas de cultivares a ambientes favorables y desfavorables y concluye que los parámetrosde estabilidad estimados describen apropiadamente la respuesta de las variedades a los sitios contrastantes donde fueron evaluados.

Algunas metodologías utilizadas para. describir la adaptación de cultivares estan basadas en agrupación de cultivares utilizando el índire de disimilaridad de Lin (Osorio 1988), sin embargo estametodologfano fue acertada al comparar el origen genéticode los cultivares agrupados, el autor concluye que loscultivares manifiestan un comportamiento semejante en las diferentes localidades, atribuible a su igualdad genética, pero esta inferencia es cierta solamente en algunos casos. Deben medirse parámetros ambientales para correlacionarlos con parámetros de estabilidad.

La eficiencia de la estimación de parámetros o modelos de estabilidad, ha sido discutida por Crossa (1988), Osorio (1988), utilizando el modelo AMMI se puede multiplicar la eficiencia de los experimentos hasta tres veces (Crossa, 1998).

\section{MATERIALES Y MÉTODOS}

El ensayo uniforme de maíz del PCCMCA involucra la evaluación de 36 híbridos de Programas Nacionales y Compafifas Privadas Nacionales y Transnacionales. Los 36 híbridos descritos en el Cuadro 1, se evaluaron bajo un diseño uniforme de látice simple, $6 \times 6$, con cuatro repeticiones en 15 localidades de Centro América, Panamá México y Estados Unidos. La parcela experimental consta de cuatro surcos de 5 metros de largo; la parcela útil es de 44 plantas teóricamente.

Las variables estudiadas fueron: rendimiento, días a flor, altura de planta y mazorca, enfermedades de importancia económica, pudrición de mazorca, cobertura de mazorca, acame y prolificidad. La responsabilidad y preparación de los ensayos se define en forma rotativa en las reuniones anuales. En 1989, Honduras fue responsable de preparación de ensayos y fueron enviados a 26 localidades, de las cuales al 15 de marzo de 1990, solo se recibieron 18 libros de campo, o sea $68 \%$ de recuperación.

\section{Análisis Estadístico}

Se realizó análisis de varianza por localidad bajo el modelo de látice para rendimiento, altura de mazorca, días a flor, mazorcas descubiertas y podridas y porcentaje de prolificidad por localidad.

El modelo está dado por:

$$
\mathrm{Y}_{\mathrm{ijq}}=\mu+\mathrm{R}_{\mathrm{i}}+\mathrm{B}_{\mathrm{ij}}+\mathrm{T}_{\mathrm{q}}+\varepsilon_{\mathrm{ijq}}
$$

Cuadro 1 Cultivares evaluados en el Ensayo Uniforme del PCCMCA en 1989.

\begin{tabular}{|c|c|c|c|}
\hline Entrada & Nombre & Origen & Color \\
\hline 1 & HR-17 & Seminal & Blanco \\
\hline 2 & H-92 & Tacsa & Blanco \\
\hline 3 & HR-15 & Seminal & Blanco \\
\hline 4 & HB-85 & ICTA, Guatemala & Blanco \\
\hline 5 & HR-10 M & Seminal & Amarillo \\
\hline 6 & H-203 & Tacsa & Amarillo \\
\hline 7 & $\mathrm{H}-90$ & Tacsa & Blanco \\
\hline 8 & B-833 & Dekalb & Blanco \\
\hline 9 & H-5 & Centa, El Salvador & Blanco \\
\hline 10 & HA-46 & ICTA,Guatemala & Amarillo \\
\hline 11 & $\mathrm{H}-9$ & Centa, El Salvador & Blanco \\
\hline 12 & H-889 & Costa Rica & Blanco \\
\hline 13 & НB-87 & ICTA, Guatemala & Blanco \\
\hline 14 & HS-UT-25 & Cuba & Amarillo \\
\hline 15 & HB-83 & ICTA & Blanco \\
\hline 16 & HS-3G3 & Cristiani Burkard & Blanco \\
\hline 17 & $\mathrm{H}-53$ & CENTA, El Salvador & Blanco \\
\hline 18 & HS-5G1 & Cristiani Burkard & Blanco \\
\hline 19 & XC-H-51 & Pioneer & Amarillo \\
\hline 20 & XC-H-53 & Pioneer & Amarillo \\
\hline 21 & HS-5G & Cristiani Burkard & Blanco \\
\hline 22 & HS-3G4 & Cristiani Burkard & Blanco \\
\hline 23 & ZCJ-66 & Pioneer & Blanco \\
\hline 24 & MAX-307 & Agridec & Blanco \\
\hline 25 & MAX-309 & Agridec & Blanco \\
\hline 26 & MAX-311 & Agridec & Blanco \\
\hline 27 & P-8822 & IDIAP, Panamá & Amarillo \\
\hline 28 & P-8812 & IDIAP, Panamá & Amarillo \\
\hline 29 & P-8802 & IDIAP, Panamá & Amarillo \\
\hline 30 & P-8941 & Dekalb & Bilanco \\
\hline 31 & H-887 & Costa Rica & Blanco \\
\hline 32 & H-B-30 & RR.NN. Honduras & Blanco \\
\hline 33 & H-B-33 & RR.NN. Honduras & Blanco \\
\hline 34 & HA-51 & RR.NN. Honduras & Amarillo \\
\hline 35 & HA-52 & RR.NN. Honduras & Amarillo \\
\hline 36 & ROCA-1 & Semillas San José & Amarillo \\
\hline
\end{tabular}

donde:

$\mathrm{Y}_{\mathrm{ijq}}=$ Efecto del q-ésimo tratamiento en el j- ésimo bloque dentro de la i-ésima repetición.

$\mu \quad=$ Efecto de la media

$\mathrm{R}_{\mathrm{i}}=$ Efecto de la repetición

$\mathrm{B}_{\mathrm{ij}}=$ Efecto del bloque incompleto

$\mathrm{T}_{\mathrm{q}}=$ Efecto del tratamiento

$\varepsilon_{\mathrm{ijq}}=$ Efecto del error

Las comparaciones de medias se realizaron por la prueba de Tukey y la Diferencia Mínima Significativa Honesta (DMSH).

\section{Análisis de Estabilidad}

El análisis combinado de estabilidad para 1989, se 
Cuadro 2 Estadísticos estimados en el Análisis de Varianza para Rendimientade 36 Hibridos de Má́z evaluados en 15 localidades del Norte, Centro América y Panamá. PCCMCA 1989.

\begin{tabular}{lllllll}
\hline País & Localidad & Código & Media & C.V. F.001 & MDS \\
\hline Honduras & Comayagua & PC01 & 4.400 & 19.00 & $* *$ & 1.280 \\
El Salvador & SC Porrillo & PC02 & 4.235 & 13.12 & $* *$ & 0.786 \\
Guatemala & Cuyuta & PC03 & 5.694 & 10.63 & $* *$ & 0.838 \\
Nicaragua & Sn. Cristobal & PC09 & 3.123 & 17.50 & $* *$ & 0.758 \\
Panamá & Parita & PC10 & 4.850 & 16.78 & $* *$ & 0.622 \\
Panamá & Los Santos & PC11 & 4.756 & $10.10^{* *}$ & 0.673 \\
Honduras & Catacamas & PC13 & 5.374 & 09.58 & $* *$ & 0.713 \\
Honduras & Omonita & PC15 & 4.620 & 12.68 & $* *$ & 0.812 \\
Honduras & Zamorano & PC16 & 5.029 & 14.45 & $* *$ & 1.080 \\
Honduras & Acacias & PC17 & 6.780 & 12.57 & $* *$ & 1.160 \\
Guatemala & Tiquizate & PC20 & 3.820 & 11.17 & $* *$ & 0.610 \\
México & Tapachula & PC21 & 5.458 & 09.53 & $* *$ & 0.721 \\
Guatemala & La Vegas & PC25 & 4.764 & 13.45 & $* *$ & 0.888 \\
México & Poza Rica & PC26 & 6.944 & 09.17 & $* *$ & 0.883 \\
USA & Weslaco & PC19 & 4.565 & 11.06 & $* *$ & 0.700 \\
& & & & & & \\
\hline
\end{tabular}

realizó bajo el modelo de Eberhart y Russell (1966).

$$
\mathrm{Y}_{\mathrm{ij}}=\mu_{\mathrm{i}}+\beta_{\mathrm{i}}+\mathrm{B}_{\mathrm{ij}}+\mathrm{I}_{\mathrm{J}}+\mathrm{S}_{\mathrm{ij}}
$$

$\mathrm{Y}_{\mathrm{ij}}=\quad$ Media varietal de la i-ésima variedad en el $\mathrm{j}$ ésimo ambiente $(1=1,2 \ldots \mathrm{v} ; \mathrm{J}=\mathrm{I}, 2,3 \ldots \mathrm{n})$.

$\mu_{\mathrm{i}}=\quad$ La media de la i-ésima variedad a través de todos los ambientes.

$\beta_{\mathrm{i}}=\quad$ Coeficiente de regresión que mide respuesta de la variedad i en varios ambientes

$\mathrm{I}_{\mathrm{J}}=\quad$ Indice ambiental obtenido como el promedio de todas las variedades en el j-ésimo ambiente menos la media general

$\mathrm{S}_{\mathrm{ij}}=\quad$ Desviación de regresión de la variedad i en el ambiente $\mathrm{j}$

Para establecer la relación existente entre la descripción de estabilidad de Eberhart y Russell(1966), se analizaron los resultados por el modelo AMMI.

$\mathrm{Y}_{\mathrm{ge}}=\mu+\alpha_{\mathrm{g}}+\beta_{\mathrm{e}}+\sum_{\mathrm{n}=1}^{\mathrm{N}} \lambda_{\mathrm{n}} \gamma_{\mathrm{gn}} \delta_{\mathrm{en}}+\rho_{\mathrm{ge}}$

donde:

$\mathrm{Y}_{\mathrm{ge}}=\quad$ es el rendimiento promedio de un genotipo $\mathrm{g}$ en ambiente e.

$\mu=\quad$ es la media general.

$\alpha_{\mathrm{g}}=\quad$ son las desviaciones de las medias de los genotipos. $\beta_{\mathrm{e}}=\quad$ desviaciones de las medias de ambientes.

$\mathrm{N}=\quad$ es el número de PCA retenidos en el modelo.

$\lambda_{\mathrm{n}}=\quad$ es el valor singular para el PCA.

$\gamma_{\mathrm{gn}}=\quad$ son los valores de vectores de los genotipos para cada PCA.

$\delta_{\text {en }}=\quad$ son los valores de los vectores para cada ambiente PCA.

$\rho_{\mathrm{ge}}=\quad$ es el residual.

\section{Análisis de Correlaciones Simples}

Para estudiar el grado de asociaciones entre rendimiento y características agronómicas de baja heredabilidad como mala cubertura y pudrición de mazorca, se estimaron coeficientes de correlación simple.

\section{RESULTADOS Y DISCUSION}

Algunos de los culti vares evaluados en catorce localidades de Centro América, Panamá y Méxicó, mostraron comportamiento relativamente diferentes, a algunos de los ambientes contrastantes donde fueron evaluados (Córdova 1978, 1989 y De Paz et al. 1979) (Cuadro 3).

En el cuadro 2 se presenta los estadísticos estimados en el análisis de varianza para rendimiento por localidad. El experimento que obtuvo el valor más bajo de CV fue la localidad de T ACSA en Tapachula, México y el valor más alto de (19.00\%) en Comayagua, Honduras. Los índices ambientales indican los contrastes de ambientes donde fueron evaluados los 36 cultivares involucrados en el presente trabajo (Cuadro 3).

En el cuadro 4 se incluye la información pertinente a los estadísticos estimados en el análisis de varianza combinado de 14 localidades, para variables de importancia económica y genética consideradas en el presente trabajo. La interacción híbrido localidad fue significativa para todas las variables lo cual indica que los genotipos mostraron una respuesta relativamente diferente en algunas localidades. La variable de mayor importancia, mazorcas podridas mostró una variación de 3.62 a $16.32 \%$ lo cual indica que existen genotipos resistentes a este factor adverso de origen biótico.

Pudrición de mazorca es la enfermedad de mayor importancia económica en Honduras, Costa Rica y Guatemala considerándose pérdidas anuales de hasta el 20\%. El híbrido HA-46 muestra una considerable resistencia a esta enfermedad (3.62\% contra 10\% de H-5 y $16.00 \%$ de Dekalb P-8941) 
Cuadro 3 Medias de rendimiento e Indices Ambientales de 36 Híbridos de Maíz evaluados en 14 Ambientes de Centro América, Panamá y México, PCCMCA 1989.

\begin{tabular}{|c|c|c|c|c|c|c|c|c|c|c|c|c|c|c|c|}
\hline $\mathrm{Hb}$ & $\mathrm{CP} 01$ & $\mathrm{CPO} 2$ & $\mathrm{CPO3}$ & $\mathrm{CPO}$ & CP10 & CP11 & CP13 & CP15 & CP16 & $\mathrm{CP} 17$ & $\mathrm{CP} 20$ & $\mathrm{CP} 21$ & $\mathrm{CP} 25$ & $\mathrm{CP} 26$ & Promedio \\
\hline 1 & 4.53 & 3.98 & 5.00 & 2.68 & 5.34 & 4.78 & 5.16 & 4.57 & 5.33 & 6.57 & 3.95 & 5.97 & 4.58 & 6.37 & 4.94 \\
\hline 2 & 5.22 & 4.54 & 5.61 & 3.46 & 5.17 & 4.65 & 5.40 & 4.32 & 5.75 & 6.41 & 3.78 & 5.34 & 4.78 & 5.91 & 5.03 \\
\hline 3 & 2.33 & 3.93 & 5.33 & 3.64 & 5.05 & 4.57 & 4.65 & 4.23 & 5.60 & 6.14 & 4.24 & 5.42 & 4.06 & 6.48 & 4.69 \\
\hline 4 & 3.88 & 4.54 & 6.41 & 4.01 & 6.57 & 5.01 & 6.49 & 5.04 & 6.01 & 8.05 & 4.50 & 6.53 & 5.51 & 7.54 & 5.72 \\
\hline 5 & 2.46 & 1.85 & 3.38 & 1.90 & 3.51 & 3.30 & 2.92 & 2.40 & 3.02 & 4.32 & 2.13 & 3.26 & 2.66 & 4.73 & 2.99 \\
\hline 6 & 3.27 & 4.00 & 4.86 & 2.42 & 4.01 & 4.69 & 4.70 & 4.41 & 4.76 & 5.03 & 3.90 & 5.40 & 5.02 & 7.33 & 4.56 \\
\hline 7 & 4.62 & 3.98 & 5.34 & 3.40 & 4.42 & 3.98 & 5.44 & 4.53 & 5.29 & 6.57 & 4.09 & 5.74 & 3.87 & 5.43 & 4.76 \\
\hline 8 & 4.56 & 3.82 & 5.39 & 3.48 & 4.82 & 4.77 & 5.80 & 4.49 & 6.39 & 7.81 & 3.51 & 5.68 & 5.07 & 7.78 & 5.24 \\
\hline 9 & 4.28 & 3.62 & 4.14 & 2.94 & 3.03 & 4.21 & 4.77 & 4.04 & 4.82 & 6.64 & 3.54 & 4.36 & 4.50 & 6.85 & 4.41 \\
\hline 10 & 6.11 & 4.63 & 6.11 & 3.66 & 4.68 & 4.70 & 5.72 & 4.11 & 5.51 & 6.83 & 3.88 & 5.66 & 4.50 & 6.82 & 5.25 \\
\hline 11 & 2.89 & 5.01 & 6.31 & 2.81 & 5.64 & 4.64 & 5.30 & 5.27 & 4.77 & 7.44 & 4.09 & 6.01 & 6.16 & 7.56 & 5.28 \\
\hline 12 & 5.63 & 4.17 & 5.97 & 3.03 & 4.39 & 4.84 & 5.98 & 4.75 & 5.54 & 6.85 & 3.77 & 5.05 & 4.08 & 7.23 & 5.10 \\
\hline 13 & 3.87 & 4.39 & 7.25 & 3.80 & 4.89 & 5.09 & 6.38 & 5.35 & 5.27 & 7.97 & 4.67 & 6.10 & 5.97 & 4.92 & 5.42 \\
\hline 14 & 3.99 & 4.03 & 4.90 & 3.15 & 4.41 & 4.37 & 4.56 & 3.77 & 4.31 & 6.66 & 3.64 & 5.26 & 4.40 & 6.34 & 4.56 \\
\hline 15 & 3.84 & 4.46 & 6.46 & 3.55 & 5.89 & 4.61 & 5.64 & 4.22 & 3.67 & 5.96 & 3.79 & 6.44 & 4.98 & 7.53 & 5.08 \\
\hline 16 & 3.56 & 4.09 & 5.96 & 2.55 & 5.09 & 5.05 & 4.99 & 5.50 & 4.09 & 6.12 & 4.15 & 6.35 & 5.75 & 7.70 & 5.07 \\
\hline 17 & 2.73 & 4.33 & 5.82 & 3.46 & 5.14 & 4.29 & 5.17 & 4.26 & 4.94 & 6.71 & 3.76 & 5.91 & 4.51 & 6.10 & 4.80 \\
\hline 18 & 3.16 & 4.21 & 6.12 & 3.22 & 4.50 & 4.41 & 5.41 & 4.41 & 4.55 & 6.07 & 3.92 & 5.76 & 4.55 & 5.54 & 4.70 \\
\hline 19 & 5.37 & 4.48 & 6.75 & 2.70 & 4.68 & 4.49 & 5.74 & 4.31 & 5.32 & 7.20 & 3.60 & 5.58 & 4.66 & 7.19 & 5.15 \\
\hline 20 & 6.05 & 4.38 & 6.05 & 3.56 & 4.51 & 4.61 & 5.18 & 5.23 & 4.91 & 7.93 & 3.74 & 5.32 & 5.65 & 7.35 & 5.34 \\
\hline 21 & 4.59 & 3.83 & 5.71 & 3.38 & 5.26 & 4.28 & 5.07 & 3.89 & 5.03 & 6.35 & 3.86 & 5.44 & 4.63 & 6.63 & 4.86 \\
\hline 22 & 5.53 & 5.16 & 6.22 & 2.69 & 5.24 & 5.20 & 5.36 & 5.34 & 4.10 & 7.15 & 4.36 & 7.25 & 5.95 & 8.36 & 5.57 \\
\hline 23 & 6.01 & 4.14 & 5.54 & 2.89 & 5.37 & 4.47 & 5.49 & 5.04 & 5.48 & 7.15 & 3.80 & 5.27 & 4.76 & 8.00 & 5.24 \\
\hline 24 & 5.97 & 4.72 & 5.44 & 2.06 & 5.18 & 5.23 & 5.18 & 4.45 & 5.17 & 6.19 & 3.70 & 5.45 & 4.47 & 7.07 & 5.02 \\
\hline 25 & 4.31 & 4.96 & 5.79 & 2.95 & 4.68 & 5.04 & 4.98 & 4.76 & 5.44 & 6.79 & 3.95 & 5.02 & 4.76 & 7.24 & 5.04 \\
\hline 26 & 5.95 & 5.33 & 6.42 & 2.60 & 5.45 & 5.25 & 5.33 & 4.88 & 5.72 & 7.46 & 4.10 & 5.38 & 4.77 & 7.86 & 5.54 \\
\hline 27 & 4.31 & 4.63 & 6.10 & 3.58 & 4.67 & 5.37 & 5.86 & 5.21 & 4.90 & 7.26 & 3.73 & 5.53 & 4.78 & 7.88 & 5.28 \\
\hline 28 & 4.42 & 4.36 & 5.80 & 2.91 & 5.37 & 5.54 & 5.06 & 5.16 & 5.84 & 7.47 & 3.37 & 6.06 & 4.78 & 7.81 & 5.29 \\
\hline 29 & 7.20 & 4.45 & 7.00 & 3.37 & 5.54 & 5.38 & 5.86 & 4.42 & 5.38 & 7.15 & 4.35 & 5.48 & 5.59 & 7.68 & 5.63 \\
\hline 30 & 6.05 & 3.83 & 5.93 & 3.17 & 4.68 & 5.35 & 7.02 & 4.57 & 5.65 & 8.03 & 3.47 & 5.13 & 4.05 & 7.32 & 5.30 \\
\hline 31 & 1.78 & 3.31 & 4.65 & 2.46 & 4.55 & 4.56 & 4.91 & 4.84 & 4.83 & 6.21 & 2.91 & 4.51 & 3.95 & 6.11 & 4.25 \\
\hline 32 & 3.24 & 4.20 & 4.97 & 3.62 & 4.38 & 5.05 & 5.63 & 5.53 & 4.61 & 7.10 & 4.20 & 5.40 & 4.79 & 7.46 & 5.08 \\
\hline 33 & 4.73 & 4.80 & 5.62 & 3.76 & 4.93 & 5.40 & 5.77 & 4.88 & 4.87 & 7.94 & 4.06 & 5.55 & 5.10 & 7.61 & 5.35 \\
\hline 34 & 3.48 & 4.12 & 5.00 & 3.11 & 4.30 & 4.68 & 5.16 & 5.07 & 4.92 & 6.38 & 3.70 & 4.49 & 4.03 & 6.65 & 4.65 \\
\hline 35 & 3.60 & 3.75 & 4.97 & 2.80 & 4.25 & 4.49 & 5.40 & 4.21 & 4.86 & 5.79 & 3.96 & 4.40 & 4.68 & 6.46 & 4.55 \\
\hline 36 & 5.03 & 4.29 & 5.56 & 2.54 & 5.41 & 4.88 & 5.34 & 4.92 & 4.11 & 6.29 & 3.29 & 4.83 & 4.57 & 7.05 & 4.88 \\
\hline Promedio & 4.40 & 4.23 & 5.69 & 3.12 & 4.85 & 4.75 & 5.37 & 4.62 & 5.03 & 6.78 & 3.82 & 5.46 & 4.76 & 6.99 & 4.99 \\
\hline$I_{j}$ & -0.59 & -0.76 & 0.70 & -1.87 & -0.14 & -0.29 & 0.38 & -0.37 & 0.04 & 1.79 & -1.17 & 0.47 & -0.23 & 2.00 & \\
\hline
\end{tabular}

este híbrido también superó, significativamente, en rendimiento al testigo H-5. (Cuadro 5). La prueba de Tukey aplicada al rendimiento discrimina en primer lugar 11 híbridos de los 36 evaluados en catorce localidades. El primer grupo lo constituyen HB-85, P-8802, HS-3G4, Max-311, H-33 y HB-87, los cuales superaron al testigo H-5 (4.80 tm/ha) con 28 a 34\%. (Cuadro 5). El HB-85 mostró adaptación a la mayoría de ambientes de prueba colocándose en los primeros cuatro lugares entre los 36 genotipos evaluados en catorce localidades (cuadro 3).

Podríamos considerar que el híbrido HB-85 llena todas las características de una variedad "deseable" (descritas por Carballo y Márquez, 1970) ya que sus características agronómicas como baja pudrición y cobertura de mazorca son excelentes. El proyecto regional de híbridos de Centro América ha logrado también notables progresos ya que el híbrido P-8802 de Panamá obtuvo también el primer lugar en rendimiento superando al testigo $\mathrm{H}-5$ en $31 \%$. Estos resultados demuestran que la colaboración horizontal puede ser un instrumento notable que permite utilizar en forma eficiente los recursos económicos y técnicos de la región. El HA-46 mantiene su liderazgo en resistencia a pudrición de mazorca lo cual incrementa su potencial en el mercado regional y apoya el enfoque de mejoramiento para resistencia a factores adversos y bióticos que en el futuro contribuirán a una producción sostenida de maíz en la región. Los coeficientes de correlaciones simples estimados en el análisis combinado indican la magnitud de la asociación existente entre el rendimiento vs. mazorcas podridas, prolificidad y acame con coeficientes $\mathrm{r}=-0.43 * *$, $0.29 *$ y $-0.45 * *$ respectivamente, mazorcas podridas vs. acame, $\mathrm{r}=0.48 * *$ (Cuadro 6).

Las diferencias entre ambientes pueden cambiar con frecuencia la magnitud en el comportamiento de un genotipo a través de diferentes localidades de prueba. Los agricultores demandan nuevos cultivares que respondan consistentemente 
Cuadro 4 Estadísticos estimados en el análisis de varianza combinado d 36 cultivares evaluados en catorce localidades de Centr América, Panamá y México. PCCMCA 1989.

\begin{tabular}{lrrrrr}
\hline & $\begin{array}{c}\text { REND } \\
\mathrm{t} / \mathrm{ha}\end{array}$ & $\begin{array}{c}\text { MAZ } \\
\text { DESC } \%\end{array}$ & $\begin{array}{c}\text { MAZ } \\
\text { POD \% }\end{array}$ & $\begin{array}{c}\text { PROL } \\
\%\end{array}$ & $\begin{array}{c}\text { ACAME } \\
\%\end{array}$ \\
\hline Media & 4.99 & 9.90 & 8.77 & 97.00 & 127.00 \\
C.V \% & 11.87 & 7.78 & 10.00 & 9.62 & 9.22 \\
MDSH & 0.642 & 5.75 & 5.29 & 9.05 & 11.80 \\
G x A & $* *$ & $* *$ & $* *$ & $* *$ & $* *$ \\
Máximo & 5.77 & 17.88 & 16.32 & 107.00 & 146.00 \\
Mínimo & 2.97 & 4.22 & 3.62 & 89.00 & 91.00 \\
\hline
\end{tabular}

Cuadro 5 Rendimiento y características agronómicas de hibrid superiores y testigos evalua dos en catorce localidades de Centr América, Panamá y México. PCCMCA 1989.

\begin{tabular}{lccccccr}
\hline Hb & Kg/ha & $\begin{array}{c}\% \text { de } \\
\text { H-5 }\end{array}$ & $\begin{array}{c}\text { D.A F } \\
\text { Fem }\end{array}$ & $\begin{array}{r}\text { Alt } \\
\text { Maz }\end{array}$ & $\begin{array}{r}\text { Maz } \\
\text { Pod } \%\end{array}$ & $\begin{array}{c}\text { Maz } \\
\text { Desc \% }\end{array}$ & $\begin{array}{r}\text { Prol } \\
\%\end{array}$ \\
\hline HB-85 & 5723 & 134 & 54 & 132 & 6.65 & 3.84 & 100 \\
P-8802 & 5608 & 131 & 55 & 124 & 7.31 & 12.31 & 106 \\
HS-3G4 & 5569 & 131 & 55 & 141 & 9.41 & 12.83 & 93 \\
MAX-311 & 5516 & 130 & 55 & 134 & 5.88 & 11.78 & 101 \\
H-B-33 & 5480 & 128 & 55 & 137 & 9.10 & 8.58 & 99 \\
HB-87 & 5431 & 128 & 55 & 129 & 7.11 & 12.58 & 101 \\
XC-H-53 & 5333 & 124 & 54 & 120 & 10.20 & 12.77 & 98 \\
P-8941 & 5286 & 124 & 57 & 130 & 16.32 & 9.44 & 102 \\
P-8812 & 5275 & 124 & 57 & 137 & 11.38 & 16.29 & 95 \\
H-9 & 5274 & 124 & 56 & 141 & 6.77 & 7.29 & 95 \\
P-8822 & 5271 & 124 & 55 & 130 & 7.65 & 7.54 & 101 \\
HA-46 & 5231 & 122 & 55 & 123 & 3.62 & 5.17 & 107 \\
B-833 & 5236 & 122 & 59 & 142 & 9.91 & 8.68 & 102 \\
H-5 & 4269 & 100 & 57 & 147 & 10.06 & 4.39 & 93 \\
& & & & & & & \\
MDSH & 602 & & 1.09 & 12 & 5.29 & 5.75 & 9 \\
CV & 8.60 & & 2.10 & 3.6 & 12.10 & 11.68 & 10.0 \\
\hline
\end{tabular}

a la mayoría de condiciones ambientales. De aquí, la necesidad de desarrollar cultivares que interaccionen positivamente con el medio ambiente utilizando modelos que permitan la identificación de genotipos estables.

El cuadro 7 presenta las medias de rendimiento Score AMMI e índices ambientales estimados en los análisis de varianza para los dos modelos de est.'lbilidad utilizados en el presente trabajo. El modelo AMMI identificó ambientes neutrales como, Catacamas, Zamorano y Acacias en Honduras. Zamorano, fue clasificado como ambiente neutral por los dos modelos. Acacias fue clasificado como ambiente rico por Eberhart y Russell.

El cuadro 8 presenta el análisis de varianza para el modelo AMMI es notable que los genotipos, ambientes e interacciones poseen el $84.5 \%$ de la suma de cuadrados total. La interacción genotipo ambiente es importante ya que captura el $50 \%$ de la suma de cuadrados de genotipos. El componente principal PCAl captura más o menos el $50 \%$ de la suma de
Cuadro 6 Coeficientes de correlación simple entre características agronómicas, PCCMCA 1989.

\begin{tabular}{|c|c|c|c|c|c|}
\hline & Rend & M. Desc & M. Pod & Prol & Acame \\
\hline Rend & & NS & $-0.43 * *$ & $0.29 *$ & $-0.45^{* *}$ \\
\hline M. Desc & & & NS & NS & NS \\
\hline M. Pod & & & & NS & $0.48 * *$ \\
\hline Prol & & & & & NS \\
\hline Acame & & & & & 1 \\
\hline
\end{tabular}

Cuadro 7 Medias de rendimiento y scores AMMI e indices ambientales. PCCMCA 1989.

\begin{tabular}{llllrl}
\hline País & Localidad & Código & Media & I A $_{\text {A }}$ & Score \\
\hline & & & & & AMMI \\
México & Tapachula & PC21 & 5.46 & 0.47 & 0.4920 \\
Honduras & Omonita & PC15 & 4.62 & -0.37 & 0.4779 \\
Nicaragua & Sn. Cristobal & PC09 & 3.12 & -1.87 & 0.4281 \\
Guatemala & Tiquizate & PC20 & 3.82 & -1.17 & 0.4280 \\
Panamá & Parita & PC10 & 4.85 & -0.14 & 0.3716 \\
Guatemala & Las Vegas & PC25 & 4.76 & -0.23 & 0.3628 \\
Panamá & Los Santos & PC11 & 4.75 & -0.29 & 0.1841 \\
El Salvador & S. C. Porrillo & PC02 & 4.23 & -0.76 & 0.1396 \\
Guatemala & Cuyuta & PC03 & 5.69 & -0.70 & 0.1112 \\
Honduras & Zamorano & PC16 & 5.03 & 0.04 & 0.0657 \\
Honduras & Catacamas & PC13 & 5.37 & 0.38 & 0.0219 \\
Honduras & Acacias & PC17 & 6.78 & 1.79 & -0.0479 \\
México & Poza Rica & PC26 & 6.99 & 2.00 & -0.1511 \\
Honduras & Comayagua & PC01 & 4.40 & -0.59 & -2.8843 \\
\hline
\end{tabular}

Cuadro 8 Análisis de varianza del rendimiento para estimar los valores aditivos de genotipos, ambientes y sus interacciones, modelo AMMI. PCCMCA, 1989.

\begin{tabular}{|c|c|c|c|c|}
\hline Fuente & G. L & \multicolumn{2}{|c|}{$\mathrm{CM}$} & $F \leq 0.01$ \\
\hline Ambientes & 13 & 163.8944 & & $* * *$ \\
\hline Genotipos & 35 & 14.8841 & & $* * *$ \\
\hline $\mathrm{G} \times \mathrm{A}$ & 455 & 1.6317 & & $* * *$ \\
\hline PCA1 & 47 & & 7.7163 & $* * *$ \\
\hline Residual & 408 & & 0.9308 & $* * *$ \\
\hline Error & 1470 & 0.5621 & & $* * *$ \\
\hline
\end{tabular}

cuadrados de la interacción indicando la importancia de $\mathrm{G}$ x A** en la estimación de los escores para los genotipos confinuando éstos resultados que el PCAI tiene valores predictivos. El residual (14.5\% de la SC total) indica que tiene posibilidades de éxito para valores predictivos, estos resultados coinciden con los obtenidos por Gauch y Zobel (1989).

La respuesta diferencial de los cultivares evaluados en diferentes ambientes implica la utilización de metodologías que penuitan discriminar adecuadamente los genotipos que contribuirán al progreso por selección. En un programa de mejoramiento, se necesita mucha precisión para seleccionar materiales superiores dentro de un grupo de genotipos cuyas diferencias en potencial de rendimiento son mínimas, por otra parte es necesario entender bien la interacción genotipo 
Cuadro 9 Medias y Parametros estimados en el Análisis de Varianza para Estabilidad del Rendimiento de 36 Híbridos de Maíz evaluados en 14 localidades. PCCMCA 1989.

\begin{tabular}{|c|c|c|c|c|c|}
\hline Nombre & $\mathrm{Kg} / \mathrm{ha}$ & $B \mathrm{i}$ & $\mathrm{Sdi}^{2}$ & $\begin{array}{l}\text { Score } \\
\text { AMMI }\end{array}$ & $r$ \\
\hline HB-85 & 5.7231 & 1.270 & 0.126 & 0.4760 & 0.780 \\
\hline P-8802 & 5.6085 & $1.590 * *$ & 0.140 & -1.0860 & 0.866 \\
\hline HS-3G4 & 5.5699 & $1.590^{* *}$ & $0.160^{*}$ & -0.2874 & 0.846 \\
\hline MAX-311 & 5.5161 & 1.268 & 0.072 & -0.5702 & 0.875 \\
\hline H-B-33 & 5.4800 & 1.280 & 0.040 & -0.0610 & 0.901 \\
\hline HB-87 & 5.4319 & 1.000 & 0.100 & 0.4675 & 0.780 \\
\hline XC-H-53 & 5.3333 & 1.155 & 0.000 & -0.8439 & 0.940 \\
\hline P-8941 & 5.2069 & $1.560^{*}$ & 0.143 & -0.7620 & 0.854 \\
\hline P-8812 & 5.2758 & $1.690^{*}$ & $0.187^{*}$ & 0.0467 & 0.830 \\
\hline $\mathrm{H}-9$ & 5.2741 & 0.993 & $0.159 *$ & 0.7925 & 0.703 \\
\hline P-8822 & 5.2717 & 1.175 & 0.040 & 0.0884 & 0.868 \\
\hline HA-46 & 5.2531 & 1.199 & 0.084 & -0.6981 & 0.850 \\
\hline B-833 & 5.2365 & 1.220 & 0.041 & -0.0654 & 0.890 \\
\hline ZCJ-66 & 5.2333 & $1.600 *$ & 0.030 & -0.6867 & 0.938 \\
\hline XC-H-51 & 5.1387 & $1.550^{*}$ & 0.020 & -0.4519 & 0.940 \\
\hline HB-83 & 5.1054 & 0.976 & 0.080 & 0.3328 & 0.800 \\
\hline H-B-30 & 5.1020 & 1.010 & 0.101 & 0.5556 & 0.784 \\
\hline $\mathrm{H}-\mathrm{S}-3 \mathrm{G} 3$ & 5.0958 & 1.099 & $0.146^{*}$ & 0.4761 & 0.754 \\
\hline H-889 & 5.0909 & 1.200 & $0.159 *$ & -0.8828 & 0.900 \\
\hline MAX-309 & 5.0215 & 1.050 & 0.106 & 0.0330 & 0.787 \\
\hline MAX-307 & 5.0175 & 1.040 & 0.040 & -0.7107 & 0.737 \\
\hline TACSA-H-92 & 5.0135 & 1.030 & 0.020 & -0.3160 & 0.890 \\
\hline HR-17 & 4.9240 & 1.270 & 0.046 & -0.0280 & 0.900 \\
\hline HS- $5 \mathrm{G}$ & 4.8666 & $1.390^{*}$ & 0.065 & -0.1021 & 0.899 \\
\hline ROCA-1 & 4.8119 & 0.999 & 0.131 & -0.3310 & 0.742 \\
\hline $\mathrm{H}-53$ & 4.8071 & 1.190 & 0.153 & 0.7391 & 0.770 \\
\hline TACSA-H-90 & 4.7487 & 0.961 & 0.080 & -0.1046 & 0.783 \\
\hline HS-5G1 & 4.7189 & 1.190 & $0.154^{*}$ & 0.5423 & 0.714 \\
\hline HR-15 & 4.6988 & $0.794 *$ & 0.050 & 0.8873 & 0.783 \\
\hline HA-51 & 4.6781 & 1.030 & 0.053 & 0.3054 & 0.852 \\
\hline HS-UT-25 & 4.5715 & 1.116 & 0.116 & 0.0688 & 0.776 \\
\hline HA- 52 & 4.5595 & 0.938 & 0.091 & 0.2255 & 0.777 \\
\hline TACSA-H-203 & 4.5512 & 1.099 & 0.070 & 0.4040 & 0.843 \\
\hline $\mathrm{H}-5$ & 4.4230 & 1.100 & 0.082 & 0.1734 & 0.830 \\
\hline H-887 & 4.2699 & 0.918 & $0.143^{*}$ & 0.9526 & 0.661 \\
\hline HR-10 M & 2.9759 & 0.640 & 0.008 & 0.2750 & 0.799 \\
\hline
\end{tabular}

ambiente para poder hacer selecciones apropiadas para una región y evitar sorpresas desagradables en el futuro (Kempton, 1984; Bradley et al., 1988, Gauch y Zobel, 1988).

El modelo AMMI ha demostrado su eficiencia por las razones siguientes: 1- Es más fácil el entendimiento de la interacción genotipo - ambiente al utilizar los Scores PCA presentados gráficamente (Kempton, 1984; Zobel el al., 1988). 2Predicción de estimaciones del rendimiento mas precisas al descartar el residual con mucho "ruido". 3- La mayor precisión se traduce en nuevas opciones para crear diseños experimentales con menos repeticiones y mayor número de tratamientos. 4-Mayor precisión mejora el éxito en seleccionar el material realmente superior ( 3 años sin usar AMMI equivalen a 2 años usando AMMI). 5- El residual del AMMI puede revelar heterogeneidad en los experimentos en el campo. 6- El mejor entendimiento de las interacciones y la mayor precisión en las estimaciones del rendimiento hacen posible las recomendaciones de variedades más confiables y mejor progreso en el programa de mejoramiento (Gauch y Zobel, 1989).
Cuadro 10 Medias de Rendimiento y Parámetros de Estabilidad de Híbridos superiores evaluados en 34 ambientes de Cento América, Panamá y México. PCCMCA 1988-1989.

\begin{tabular}{lcccc}
\hline Híbrido & $\mathrm{Kg} / \mathrm{Ha}$ & $\% \mathrm{H} 5$ & $\mathrm{Be}$ & $\mathrm{S}_{\mathrm{di}}{ }^{2}$ \\
\hline HB-85 & 6077 & 128 & $1.05 \mathrm{NS}$ & $0.110 \mathrm{NS}$ \\
HB-87 & 6066 & 128 & $1.00 \mathrm{NS}$ & $0.090 \mathrm{NS}$ \\
HB-83M & 5924 & 125 & $0.97 \mathrm{NS}$ & $0.070 \mathrm{NS}$ \\
MAX-307 & 5764 & 122 & $1.15 \mathrm{NS}$ & $0.135 * *$ \\
HA-46 & 5709 & 121 & $1.19 * *$ & $0.070 \mathrm{NS}$ \\
B-833 & 5662 & 120 & $1.22 * *$ & $0.030 \mathrm{NS}$ \\
XC-H-53 & 5461 & 115 & $1.15 \mathrm{NS}$ & $0.000 \mathrm{NS}$ \\
H-5 & 4731 & 100 & $1.10 \mathrm{NS}$ & $0.070 \mathrm{NS}$ \\
\hline
\end{tabular}

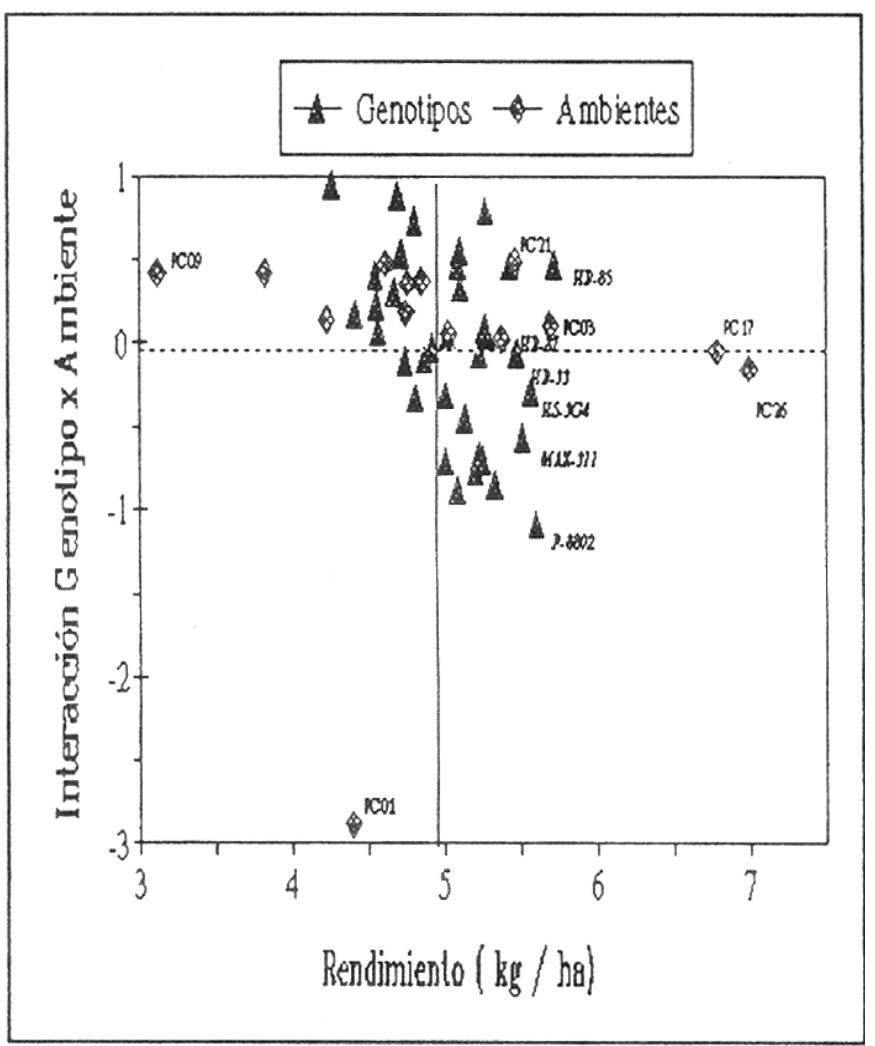

Figura 1 Estabilidad de híbridos de Maíz evaluados en 14 ambientes de Centro América y Panamá. Modelo AMMI. 1989.

Los cuadros 9 y 10 presentan los parámetros de estabilidad estimados para el análisis de varianza de estllbilidad de rendimiento 1989 y el combinado de 32 localidades, 2 años y (36 repeticiones) respectivamente. El modelo de Eberhart y Russell identificó a los híbridos HB-87, HB-85 y XC-H-53 como los más estlbles $\left(\beta=1, \mathrm{~S}_{\mathrm{di}}{ }^{2}=0\right)$. Los híbridos más estlbles identificados por el modelo AMMI fueron HB-33, HS-3G4, MAX-309, B-833 (Figura 1), P-8802 de Panamá, este último híbrido fue clasificado con respuesta superior a ambientes ricos $\left(\beta_{\mathrm{i}}=1.59 * *\right)$ por Eberhart $\mathrm{y}$ Russell (1966). 
El híbrido HB-85 fue clasificado como un híbrido que responde bien a la mayoría de ambientes. Los dos modelos coinciden solamente en el $40 \%$ de la identificación de híbridos que interaccionan menos con el ambiente. La mayoría de genotipos identificados como estables por el AMMI estuvieron abajo de la media de rendimiento. Loshíbridos HB-85, HB-87, HB-83 y XC-H-53 mantienen su estabilidad del rendimiento a través de años y localidades con rendimientos de $6,000 \mathrm{~kg} / \mathrm{ha}$ superando al testigo H-5 hasta en un $28 \%$ de rendimiento, MAX 307, B-833 y HA-46 mostraron poseer inconsistencia en su respuesta pero superaron al testigo $\mathrm{H}-5$ hasta con $21 \%$.

\section{CONCLUSIONES Y RECOMENDACIONES}

1. El nuevo híbrido HB-85 rindió $5723 \mathrm{~kg} /$ ha superando al testigo $\mathrm{H}-5$ con $34 \%$ y con características agronómicas de cobertura y pudrición de mazorca excelentes. Su adaptación a la mayoría de ambientes en Centro América, Panamá y México fue notable. En forma similar se comportó P-8802, HB-87, P-8812, XC-H-53, MAX 311 y HB-33.

2. Se ha realizado un notable progreso en el desarrollo de híbridos en los 3 años pasados y la mayoría de los programas nacionales poseen híbridos superiores al testigo, sin embargo, falta una promoción agresiva para que estos genotipos lleguen a los agricultores.

3. El modelo de Eberhart y Russell y AMMI coincidieron solamente en un $40 \%$ en la identificación de genotipos estables y con media de rendimiento alta a través de los diferentes ambientes.

4. El análisis de combinado de est11bilidad de 34 localidades en 2 años y 136 repeticiones identificó a los híbridos HB-85, HB-87, HB-83M y XC-H-53 como híbridos estables $\left(\beta \mathrm{e}=1, \mathrm{~S}_{\mathrm{di}}{ }^{2}=0\right)$ con rendimiento de 6.00 ton/ha superando al testigo $\mathrm{H}-5$ en $28 \%$.

5. Se recomienda que se impulsen programas agresivos de transferencia de tecnología y de producción de semilla de buena calidad. Estableciendo programas de transferencia masiva.

\section{BIBLIOGRAFIA}

ALLARD, R. W.; BRADSHAW. 1967. Implication of Genotype environment interaction, in applied plant breeding. Crop Sci. 4;503-509.

BONNY, R.N., Tate. ;M. KERLOVA. 1985. Segregating Populations of Cow Pea. Crop ScL 25. 208-210.

BLUM, A. 1988. Plant breeding for stress environments. 18-28. CRC press.
BRADLEY J.P., KNITTLE K.H., TROYER A.F. 1988. Statistical methods in seed coro production and selection. J. Prod Agric 1:34-38

CARBALLO, C.A., y MARQUEZ, S.P. 1970. Comparación de variedades de maíz de El Bajio y La Mesa Central por su rendimiento y estabilidad. Agro-Ciencias 5 (1): 129-146.

CORDOV A, H.S. 1978. Uso de parámetros de estabilidad para evaluar el comportamiento de variedades. Guatemala, ICTA 35 p.

CORDOVA, H.S., RAUN, W., y BARKER. T. 1988. El usodeparámetrosde estabilidad para determinar la adaptación de 36 cultivares de 'maíz. Simposium Modelos de Estabilidad para Evaluar la Adaptación de CuItivares. XXXIV Reunión Anual del PCCMCA. San José, Costa Rica.

CORDOVA, H.S. 1989. Evaluación de 36 cultivares de maíz en 20 ambientes de Centro América, Panamá y El Caribe PCCMCA. XXXV Reunión Anual del PCCMCA, San Pedro Sula. Honduras, abril 2 al 7.

CROSSA, J. 1988. Análisis predictivo y posdictivoen evaluación de cultivares de maíz. Simposio, uso de modelos de estabilidad para determinar la adaptación de cultivares. XXXIV Reunión Anual del PCCMCA, San José, Costa Rica.

DA VILA. P.A., CORDOV A, H.S., y POEY F.R. 1978. Uso de parámetros de estabilidad en la evaluación de variedades comerciales y experimentales de maíz (Zea mays L): (I) Zona Media. XXIV Reunión Anual del PCCMCA, San Salvador.

DE PAZ, R., POEY F., y CORDOVA. H.S. 1977. Uso de parámetros de estabilidad para evaluar el comportamiento de variedades criollas de maíz (Zea mays L.) en el altiplano de Guatemala (II) zona alta. XXIV Reunión Anual PCCMCA, San Salvador, C.A.

DE PAZ, R., OZAETA M. y CORDOVA, H.S. 1979. Segunda fase en la Evaluación de variedades criollas de maíz (Zea mays L.) en el altiplano de Guatemala. XXV Reunión Anual PCCMCA, Tegucigalpa, Honduras, C.A.

EBERHAR T. S.A.; RUSSELL. W.A. 1966. Stability parameters for comparing varieties. Crop. Sci. 6:36-40

FINLAY, K.W.; WILKINSON. G.N. 1963. The analysis of adaptation in plant breeding program, Aust. J. of Agriculture research, 14,742.

GADNER. C.O. y MARECK. J.H. 1977. Stability of yield of original and improved populations of maize grown over a wide range oren vironments. Agron. Abst. 55p. Am. Soc. Agron.

GAUCH, H.G., ZOBEL, R.W. 1988. Predictive and postdictive success of statistical analysis of yield trials. Theory Appl Genet 76:1 10 Gauch. H.G. and Zobel, R.W. 1989. Accuracy andselectionsuccessin yieldtrial analyses. Theor Appl. Genet 77:473-481.

KANG, M.S. 1988 B. A rank-sum method for selectioll high yielding, stable corn genotypes. Cereal Res. comun. 16: 113-115.

KANG. M.S. 1990. Understanding and utilitation of genotipe by enviroment interaction in plant breeding. Symphosium in : Genotipe by enviroment interaction and plant breeding. Lousiana State University, Agricultural Center. Page 65.

KEMPTON, R.A.1984. The use of biplots in interpreting variety by environment interactions. J Agric Sci 103:123-135

MARQUEZ, S.F.. VALLEJO. R.P. y CORDOVA, H.S. 1983. Variedades sintéticas de maíz. Colegio de Post-Graduados, Chapingo. México 70p. 
MIEZAN, K., WALKER, T.L., MILIKEN, G.A.,y LIAN, G.H. 1977. Problerns in using regression coefficients as stability parameters in breeding programo Agron. Abst. 64p. Am. Soc. Agron.

OSORIO, F. 1988. Análisis predictivo y posdictivo en evaluación de cultivares de maíz. Simposio. uso de modelos de estabilidad para determinar la adaptación de cultivares. XXXIV Reunión Anual del PCCMCA. San José. Costa Rica.

SAlGuERO, V.. CORDOVA, H.S., CRISOSTOMO. C. y POEY, F.R. 1977. Uso deparámetros de estabilidad en la evaluación de híbridos comerciales y experimentales de maíz (Zea mays L): XXII Reunión Anual del PCCMCA. Panamá.

SHUKLA, G.K. 1972. Some statistical aspects of partitioning genotype enviromental components of variability. Heredity 29: 237-245.

ZOBEL, R.W.; WRIGHT, M.J.; GAUCH, H.G. 1988. Statistical analysis of a yield trial. Agron. J. 80:388-393

\section{RECONOCIMIENTO}

El presente documento es el resultado del trabajo cooperativo, de las siguientes entidades e instituciones de Centro América, Panamá, El Caribe y México; a quienes se agradece por su invaluable aportación profesional.

Costa Rica Kenneth Jiménez, Carlos Salas, (Universidad de Costa Rica), José González, Carlos Calderón (Ministerio de Agricultura).
El Salvador Adán Aguiluz, Héctor Deras (CENTA).

Guatemala Carlos Pérez, Luis Larios, Nery Soto, José Luis Quemé (ICTA); Antonio Cristiani (Cristiani Burkard); Roberto Velázquez (SEMINAL, TACSA)

Honduras Luis Brizuela, Víctor Méndez, Humberto Mejía, José Paz, (S.R.N.); Leonardo Corral (El Zamorano).

Nicaragua Roger Urbina, Marvin Ovando (MIDINRA)

México Semilla TACSA, Ramón J. Godoy (Semillas Híbridas. S.A.): PIONEER (Selecciones Genéticas).

Rep. Dom. Ramón Celado. Rodolfo Pierre (CESDA).

Panamá Alfonso Alvarado, Daniel Pérez, Ismael Camargo (IDIAP)

E.E.U.U Federico Poey (AGRIDEC).

** Los coordinadores de los Programa Nacionales de Maíz (CRP) agradecen a las compañías de semillas que operan en la región, por el apoyo económico brindado para la conducción de los experimentos, siendo estas:

DEKALB, CRISTIANI BURKARD, SEMINAL, PIONEER, AGRIDEC y SEMILLAS TACSA. 